\title{
DISEÑO Y PUESTA EN FUNCIONAMIENTO DE UN SIG COMO HERRAMIENTA PARA EL ESTUDIO DEL TURISMO Y SU PLANIFICA- CIÓN EN LAS REGIONES DEL ARCHIPIÉLAGO DE LAS CANARREOS Y CIENFUEGOS-TRINIDAD-TOPES DE COLLANTES, CUBA.
}

\author{
por \\ QUINTELA FERNANDEZ, J.; REMONDI NOA, R. Y DURÁN GREGORIO, M.E. \\ (Facultad de Geografia. Universidad de La Habana)
}

\section{RESUMEN}

En la época actual, los Sistemas de Información Geográfica están siendo cada vez más utilizados como plataforma para el manejo de grandes volúmenes de información relacionados con la toma de decisiones en diferentes ramas. Dada la gran variedad de recursos turísticos con que Cuba cuenta y dado el incremento de la actividad en el país, se han generado por la Facultad de Geografia de la Universidad de La Habana diferentes sistemas orientados al estudio y la planificación del turismo. En el presente trabajo se presentan dos de estos resultados vinculados con territorios específicos: El Archipiélago de los Canarreos y la región Cienfuegos - Trinidad - Topes de Collantes.

Palabras Claves: Sistema de Información Geográfica, capas temáticas, datos espaciales.

\section{ABSTRACT}

Today, geographic information systems are being used as a platform for managing hugh volumes of information related to the decision making process within different fields. Given the great variety of touristic resources that Cuba has, different systems have been generated at the Faculty of Geography of the University of Havana, oriented to tourism planning and study. In this article, two of those results are presented involving different territories: Los Canarreos Archipielago and Cienfuegos Trinidad - Topes de Collantes region.

KEYwORDS: Geographic Information Systems, thematic layers, spatial data.

\section{INTRODUCCIÓN}

En muchos países el turismo constituye un recurso y una vía para su desarrollo, por lo que su planificación resul- 
ta una tarea de una gran complejidad y esta debe realizarse teniendo en cuenta todo un conjunto de elementos y recursos, (transporte, alojamiento, hoteles, restaurantes, redes viales, recursos naturales, fuerza de trabajo, etc.) que deben analizarse de manera integrada. Este cúmulo de información puede ser en gran parte almacenada, recuperada, $y$ gestionada de manera muy eficiente por los Sistemas de Información Geográfica.

En el mundo actual pocas esferas se encuentran fuera del alcance de estas herramientas tecnológicas que agilizan y hacen mucho más confiables los análisis en términos espaciales (cálculos de áreas, distancias, volúmenes, alturas, etc.), temáticos (cantidad de población, tipos de suelos presentes, tipos de arena en playas, etc.) y mixtos. De acuerdo a este último aspecto es posible hallar respuesta a las siguientes preguntas:

¿ Dónde localizar un hotel que cumpla con ciertos requerimientos técnicos, que se encuentre cerca o sobre el recurso a explotar y que al mismo tiempo pueda estar al acceso de la fuerza de trabajo, de forma tal que sean mínimos sus movimientos pendulares?

$¿$ Por donde establecer una ruta por carretera para turismo en grupo que satisfaga las expectativas que se identificaron durante los estudios de mercado y de comportamiento ante decisiones?
Estos no son los únicos aspectos en los que se obtendrían ventajas del uso de los SIG, no debería reducirse su aplicación a cuestiones vinculadas solamente a la localización; también pueden emplearse en general como apoyo al diseño de nuevos productos turísticos, a la visualización y estudio de flujos de visitantes, a análisis estadísticos de diverso tipo, a la elaboración de salidas cartográficas y otros. documentos publicitarios, a estudios de impacto, de demanda, entre otros.

\section{DISEÑO DEL SIG}

En este trabajo se pretende proponer el diseño, el contenido, la estructura y algunas de las funciones que podrán ser ejecutadas en un Sistema de Información Geográfica aplicado al estudio del turismo en la regiones turísticas Archipiélago de los Canarreos y Cienfuegos - Trinidad - Topes de Collantes, que les permita a los planificadores e investigadores del turismo encontrar solución a problemas relacionados con esta esfera, mediante la búsqueda y el manejo de la información geográfica y turística de forma rápida y eficaz.

El enfoque que conducirá el desarrollo de esta trabajo a partir de ahora esta influido por la metodología de diseño de sistemas, sin embargo, esto no implica que se pretenda abarcar 
todos los aspectos que esta incluye en su posible relación con el turismo.

Interrogantes como: ¿Que mapas debe producir el sistema? ¿Qué datos serán accesibles desde el sistema?, ¿Quién actualizaría los datos y cómo las actualizaciones se diseminarán entre los usuarios?. ¿Qué software y qué hardware son necesarios?, ¿Que personal técnico será necesario para operar el sistema?, entre otras, pueden ser respondidas en el proceso del diseño de sistemas (Huxhold W. \& Levinsohn A., 1995).

Como nuestro objetivo esta dirigido al diseño del contenido, la estructura y algunas de las funciones que podrán ser ejecutadas y no al diseño completo a la medida de un grupo u organización en específico, lo cual implicaría muchísimos más aspectos, solamente se profundizará en:

- Determinar que información debe contener el sistema y como debe ser organizada

- ¿Que Software y Hardware deben ser empleados?

- Puesta en funcionamiento del SIG.

Un aspecto muy importante para el diseño de un SIG de tal naturaleza, es la definición del contenido (nivel conceptual) de una Base de Datos Geográfica aplicada al estudio y la planificación del Turismo.
De acuerdo a la metodología ANSISPARC (American National Standards Institute, Standards Planning and Requirements Comitee), existen cuatro niveles diferentes para el modelamiento de datos que se pueden tomar como etapas para el diseño de Sistemas de Información a partir de la percepción del llamado mundo real, estos son ( en Laurini \& Thompson, 1992):

- Nivel externo

- Nivel conceptual

- Nivel lógico

- Nivel interno

De acuerdo con los objetivos de este trabajo, solamente resulta de interés el cómo definir los modelos externos y el modelo conceptual para la base de datos a crear.

Los modelos externos responden por un lado a la percepción que del mundo real tienen diferentes grupos de usuarios $u$ organizaciones, y por otro a las necesidades de información, vinculadas a cada uno de los procedimientos de análisis y recuperación necesarios para la toma de decisiones.

Este proceso se cumplimentó mediante la aplicación de cuestionarios a diversos grupos o usuarios vinculados con el estudio y la planificación del turismo, con el objetivo de hacer un inventario de sus necesidades. Diversos modelos son sugeridos para cumplimentar esta 
tarea, en este caso fue necesario crear un modelo propio (figura 1).

Acompañando a estos cuestionarios se generaron descripciones para cada uno de los mapas mencionados acerca de elementos como la escala, la precisión, la fuentes de información básica, el año de creación y las fechas de actualización y sus motivos, entre $\mathrm{Al}$. aspectos. Entre los organismos e instituciones encuestadas se encuentran:
- Instituto de Planificación Física Nacional

- Dirección Provincia de Planificación Física de Cienfuegos

- Cadena Turística Horizontes Hoteles

- Empresa Flora y Fauna (Villa Clara)

- Grupo de Investigación "Geoecología y Turismo" Facultad de Geografía. Universidad de la Habana

Figura 1.Cuestionario aplicado

\section{Cuestionario}

Fecha

Organismo :

\section{Departamento o Área:}

Misión (objetivo principal):

\begin{tabular}{|c|c|c|c|c|c|}
\hline Tareas & Objetivos & Mapas Usados & $\begin{array}{c}\text { Fuente, } \\
\text { escala, año }\end{array}$ & $\begin{array}{c}\text { Procedimientos } \\
\text { utilizados }\end{array}$ & Resultados \\
\hline & & & & & \\
& & & & & \\
\end{tabular}

\begin{tabular}{|c|c|c|c|}
\hline Otros Datos Usados & Fuente & Problemas actuales con los datos & $\begin{array}{c}\text { Necesidades } \\
\text { Futuras }\end{array}$ \\
\hline & & & \\
& & & \\
\hline
\end{tabular}


Como resultado de estos cuestionarios se procedió a la creación de una matriz en la cual se plasmaron las principales necesidades compartidas por las instituciones encuestadas (posibles usuarios del SIG). Para poder definir los elementos espaciales y los atributos a tener en cuenta en el SIG y definir el modelo conceptual a utilizar. En este caso fue seleccionado el Modelo Vectorial

Una vez definidos ambos elementos se determinó el hardware mínimo necesario:

- Una computadora personal Pentium, con $16 \mathrm{Mb}$ de RAM.

- Un monitor Super VGA.

- Una impresora.

Esta plataforma no es necesariamente la que se utilizó por el grupo de trabajo para diseñar e implementar el SIG, sino la que requerirían las entidades que harán uso de él.

\section{REGIONES DE ESTUDIO}

En el estudio sobre el potencial turístico de Cuba realizado por el Departamento de Planeamiento Turístico del Instituto de Planificación Física, se determinaron distintas regiones turísticas del país a partir de las potencialidades en los recursos que existieran. Dos de estas regiones o Polos Turísticos son: El Archipiélago de los
Canarreos y la Región Cienfuegos Trinidad - Topes de Collantes

\section{ARCHIPIÉLAGO DE LOS CANARREOS}

Esta región comprende parte del archipiélago del mismo nombre, en específico los territorios pertenecientes al grupo insular llamado Jardines y Jardinillos, que se localizan al sur de la Isla de Cuba. Este grupo insular presenta una orientación de Oeste a Este desde la Isla de la Juventud con $2200 \mathrm{Km}^{2}$ de superficie (la segunda en tamaño del Archipiélago Cubano), hasta Cayo Largo con $37 \mathrm{Km}^{2}$.

La belleza de los paisajes terrestres y marinos de esta región, la buena conservación de sus recursos florísticos y faunísticos, su acuatorio circundante y los numerosos atractivos entre los que se encuentran playas, arrecifes coralinos, fondos submarinos, e incluso residuarios aborígenes y pinturas rupestres, le confieren una connotación relevante en el Caribe.

El centro poblacional mas importante en la región se encuentra en el Norte de la Isla de la Juventud, con una población de 44519 habitantes.

\section{REGIÓN CIENFUEGOS-TRINIDAD-TOPES DE COLLANTES}

Esta otra región turística se localiza al sur de la provincias centrales de Cuba, abarcando un área aproximada- 
mente de $2320 \mathrm{Km}^{2}$.La misma se extiende sobre los municipios: Cienfuegos, Cumanayagua, Trinidad y Manicaragua en las provincias: Cienfuegos, Sancti Spiritus y Villa Clara respectivamente, donde existen centros urbanos de gran importancia económica y turística, como las ciudades de Cienfuegos y Trinidad, esta última una ciudad Colonial fundada a principios del siglo XVI

Una porción significativa de esta área presenta un relieve montañoso perteneciente al Macizo de Guamuhaya , con una altura media de 600-700 m sobre el nivel del mar, donde existe una densa red hidrográfica con la presencia de embalses. La región presenta un gran interés desde el punto de vista natural, destacándose por la abundancia de saltos de agua, pozas, miradores naturales y paisajes de gran belleza entre Al. atractivos.

\section{I.2 CONTENIDO DE LA BASE DE DATOS ESPACIALES}

Para poder llevar a cabo la puesta en funcionamiento del SIG en las dos regiones, uno de los resultados intermedios fue la determinación del contenido de la base de datos espaciales y no espaciales o de atributos. Esto implicó en una primera instancia el establecimiento de capas o layers, que definen conjuntos de información temáticamente homogénea con dimensiones topológicas similares.

Para los territorios o Polos turísticos y a partir del resultado de los cuestionarios aplicados, se definieron un conjunto de 12 capas temáticas: Región, Municipios, Vias, Asentamientos, Drenaje, Manzanas, Hoteles, Atractivos Turísticos, Playas, Cuevas y Servicios.

Para garantizar las funciones analíticas y la búsqueda de información fue necesario en cada una de las capas contar con información asociada (atributos). A continuación se relaciona alguna de esta información para las capas antes descritas.

Una vez conocidos los elementos espaciales y sus atributos, se pasó entonces a la etapa de puesta en funcionamiento. 


\section{Capa Región}

\begin{tabular}{|l|l|l|l}
\hline ID (ldentificación & Superficie \\
\hline
\end{tabular}

Capa Asentamientos

\begin{tabular}{|l|l|l|l|l|l|l|l|}
\hline ID & Nombre & Área & Tipo & Unidad & Rango Administrativo & Población & Densidad de Población \\
\hline
\end{tabular}

\section{Capa Hoteles}

\begin{tabular}{|l|l|l|l|l|l|l|l|}
\hline ID & Nombre & $\begin{array}{l}\text { Tipo de Instala- } \\
\text { ción }\end{array}$ & Habitaciones & Dirección & Teléfono & Servicios & Especialidad \\
\hline
\end{tabular}

Capa Playas

\begin{tabular}{|c|c|c|c|c|c|c|c|c|}
\hline ID & Nombre & Municipio & Longitud & $\begin{array}{c}\text { Franja de Sol } \\
\text { (anchura) }\end{array}$ & $\begin{array}{l}\text { Forma del } \\
\text { litoral }\end{array}$ & $\begin{array}{l}\text { Color de la } \\
\text { arena }\end{array}$ & $\begin{array}{l}\text { Tipo de } \\
\text { fondo }\end{array}$ & Granulometria \\
\hline
\end{tabular}

\section{Capa Cuevas}

\begin{tabular}{|l|l|l|l|l|l|l|}
\hline ID & Nombre & Municipio & Coordenadas & Largo (m) & Altura (m) & Recorrido interno \\
\hline
\end{tabular}

\section{PUESTA EN FUNCIONAMIENTO DEL SIG}

Dada la extensión de los territorios y el volumen de información a utilizar fueron seleccionadas las escalas 1:100 000 para el caso del Archipiélago Los Canarreos y 1:
50000 para la Región Cienfuegos Trinidad Topes de Collantes como escalas de entrada de Información al SIG

Este proceso se puede resumir en el siguiente esquema: 
Figura 2. Etapas seguidas para la puesta en funcionamiento del SIG.

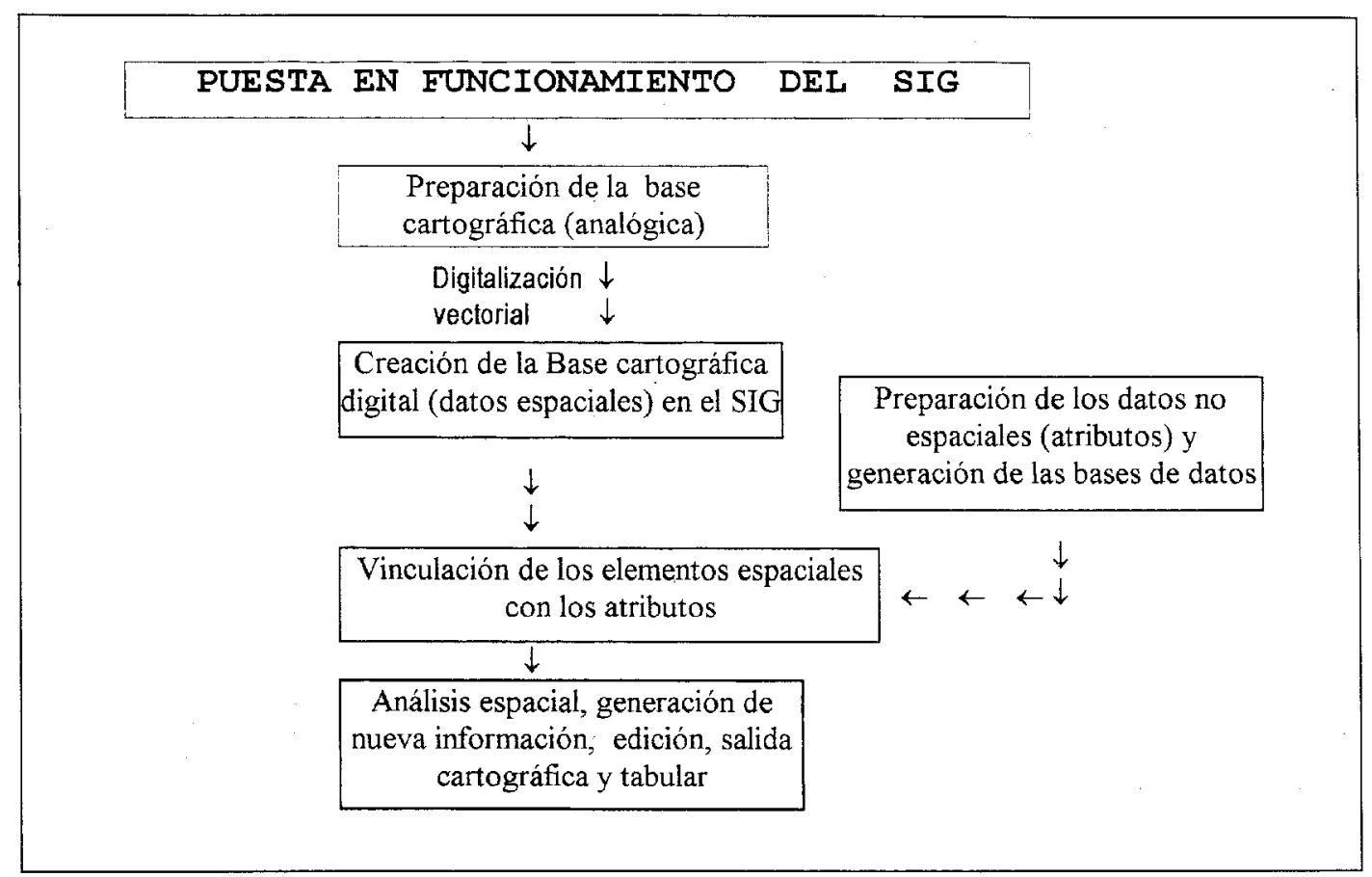

A partir de la creación de las capas mencionadas anteriormente y su vinculación con los atributos, fue posible el manejo de la información dentro del SIG con vistas a la solución de problemas relacionados con las regiones turísticas estudiadas. En la figura 3 se presentan dos ejemplos gráficos que muestran algunas de las posibilidades del SIG creado para la región de Cienfuegos Trinidad - Topes de Collantes

\section{CONSIDERACIONES FINALES}

El uso de los sistemas de información geográfica para el estudio y la pla- nificación del turismo en las regiones: Archipiélago de los Canarreos y Cienfuegos-Trinidad-Topes de Collantes constituye una herramienta de gran poder al permitir el manejo de la información y el cumplimiento de variadas operaciones de análisis de forma rápida y eficiente.

La aplicación de los cuestionarios a los posibles usuarios del sistema constituyó un método efectivo en la determinación del contenido de las bases de datos, las funciones y el software y hardware del SIG 
La Base de Datos quedó estructurada en 12 capas de información espacial: (Municipios, Asentamientos, Lagunas Región, Manzanas, Ríos, Vías, Atractivos Turísticos, Servicios, Cuevas, Playas y Hoteles); cada una con información no espacial asociada.
Con el SIG implementado se pueden generar salidas informáticas altamente eficientes que garantizan dar una respuesta rápida y precisa a los problemas planteados garantizando continua actualización de la información. 
Figura 3. Algunos ejemplos de funcionamiento del SIG para la región CienfuegosTrinidad-Topes de Collantes.

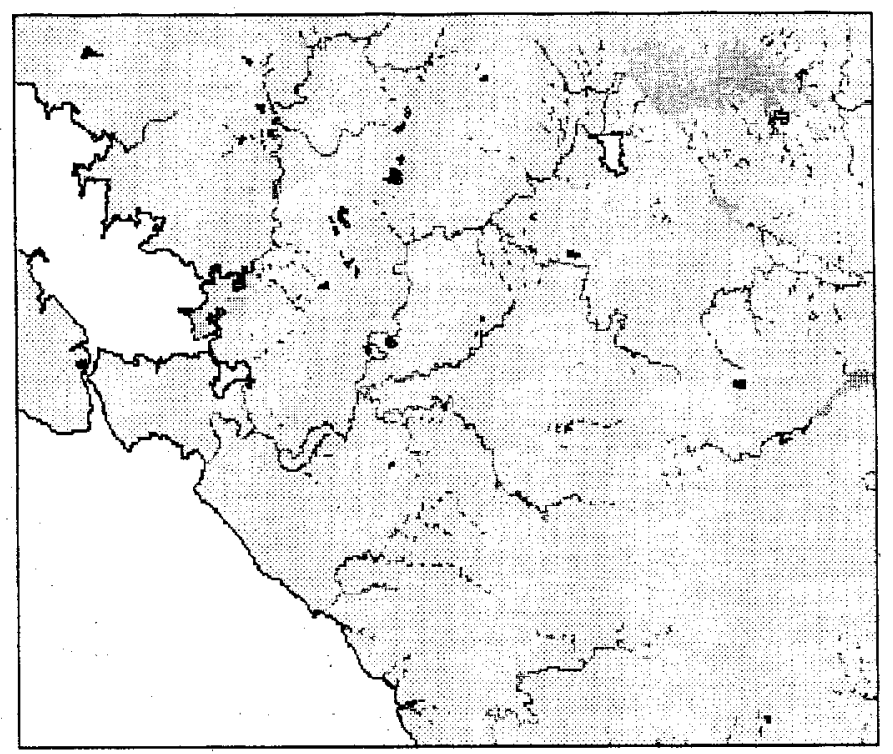

3a. Región Cienfuegos-Trinidad-Topes de Collantes (escala aproximada 1:440,000), capas activas: región y drenaje)

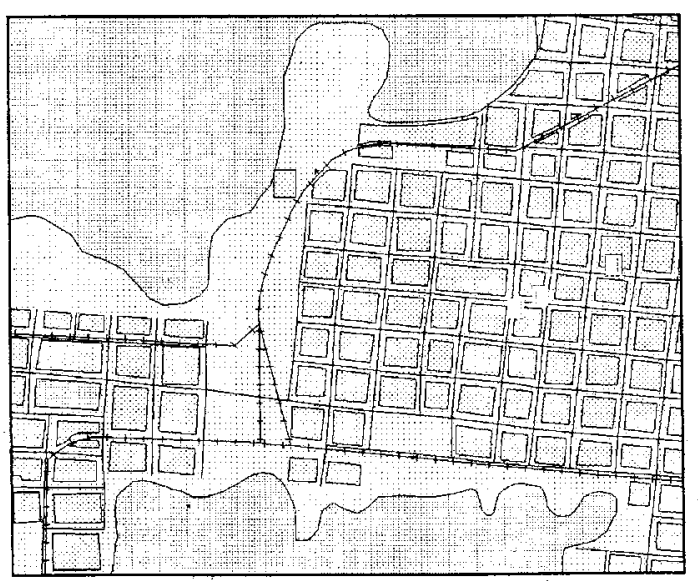

3b. Región Cienfuegos-Trinidad-Topes de Collantes (escala aproximada 1:20,000), capas activas: región, manzanas, vías y hoteles) 